\title{
Placental Stem Villus Arterial Remodeling Associated with Reduced Hydrogen Sulfide Synthesis Contributes to Human Fetal Growth Restriction
}

\author{
Liangjian Lu, ${ }^{* \dagger}$ John Kingdom, ${ }^{\ddagger}$ Graham J. Burton, ${ }^{*}$ and Tereza Cindrova-Davies*
}

From the Centre for Trophoblast Research, * University of Cambridge, Cambridge, United Kingdom; the Khoo Teck Puat-National University Children's Medical Institute, ${ }^{\dagger}$ National University Hospital, National University Health System, Singapore, Singapore; and the Mount Sinai Hospital, ${ }^{\ddagger}$ University of Toronto, Toronto, Ontario, Canada

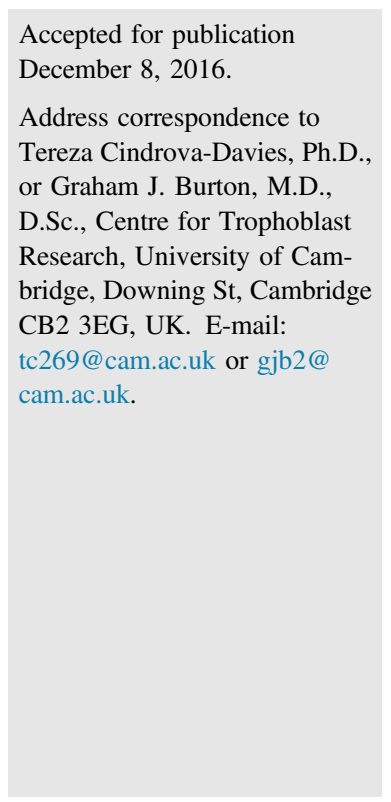

\begin{abstract}
Intrauterine fetal growth restriction (IUGR) is often associated with compromised umbilical arterial flow, indicating increased placental vascular resistance. 0xidative stress is causatively implicated. Hydrogen sulfide maintains differentiated smooth muscle in vascular beds, and its synthetic enzyme cystathionine$\gamma$-lyase (CSE) is down-regulated in growth-restricted placentas. We hypothesized that remodeling of resistance arteries in stem villi contributes to IUGR by compromising umbilical blood flow via oxidative stress, reducing hydrogen sulfide signaling. Stem villus arteries in human IUGR placentas displaying absent or reversed end-diastolic flow contained reduced myosin heavy chain, smooth muscle actin, and desmin, and increased markers of dedifferentiation, cellular retinol-binding protein 1, and matrix metalloproteinase 2, compared to term and preterm controls. Wall thickness/lumen ratio was increased, lumen diameter decreased, but wall thickness remained unchanged in IUGR placentas. CSE correlated positively with myosin heavy chain, smooth muscle actin, and desmin. Birth weight correlated positively with CSE, myosin heavy chain, smooth muscle actin, and desmin, and negatively with cellular retinolbinding protein 1 and matrix metalloproteinase 2. These findings could be recapitulated in vitro by subjecting stem villus artery explants to hypoxia-reoxygenation, or inhibiting CSE. Treatment with a hydrogen sulfide donor, diallyl trisulfide, prevented these changes. IUGR is associated with vascular remodeling of the stem villus arteries. 0xidative stress results in reduction of placental CSE activity, decreased hydrogen sulfide production, and smooth muscle cell dedifferentiation in vitro. This vascular remodeling is reversible, and hydrogen sulfide donors are likely to improve pregnancy outcomes. (Am J Pathol 2017, 187: 908-920; http://dx.doi.org/10.1016/j.ajpath.2016.12.002)
\end{abstract}

Up to $15 \%$ of pregnancies worldwide exhibit intrauterine fetal growth restriction (IUGR). The adverse effects are manifested not only by increased perinatal complications, morbidity, and mortality, but also by long-lasting consequences, including an increased risk of cardiovascular disease, hypertension, dyslipidemia, obesity, and type 2 diabetes mellitus, for the offspring. ${ }^{1-5}$ Most (65\% to $70 \%$ ) pregnancies complicated by severe IUGR typically exhibit abnormal umbilical artery Doppler waveforms indicative of increased vascular resistance, ${ }^{6}$ with the most severe cases being accompanied by absent, or even reversed, enddiastolic flow. ${ }^{7}$ Such waveforms are highly predictive of fetal hypoxemia and metabolic compromise. ${ }^{8}$ This finding supports the widely accepted hypothesis that placental insufficiency secondary to increased placental vascular resistance is the pathological process underlying most noninfective cases of IUGR ${ }^{6,7,9,10}$ in high-income countries, whereas nutritional deficiency might be a greater contributor to IUGR in low-income countries.

The key functional unit of the placenta is the villus tree, which forms the interface with the maternal blood. Stem villi arise from the chorionic plate, and their repeated

Supported by the Wellcome Trust grant 084804/2/08/Z (G.J.B.).

G.J.B. and T.C.-D. contributed equally as senior authors.

Disclosures: None declared. 
branching ultimately forms terminal villi, which are the site of maternal-fetal exchange. There is a need for unimpeded blood flow in both circulations to perform this function efficiently. Fetal blood is carried to the villi from the umbilical arteries, via chorionic arteries, to stem villus arteries (SVAs). After circulating through the villus capillaries, the blood is returned to the fetus by the umbilical vein. The SVAs are resistance vessels that determine blood flow to various parts of the placenta to match maternal perfusion. By contrast, maternal blood is delivered into the placenta from the uterine spiral arteries. These undergo remodeling in early pregnancy to ensure a high volume of blood flow, but at a low velocity to avoid damage to the delicate villus tree. Failure of remodeling is associated with IUGR and preeclampsia, when the resultant malperfusion is believed to induce placental oxidative stress. ${ }^{11,12}$

Total fetoplacental vascular impedance is determined by the structure of the arterial tree. In terms of active tension, the fetoplacental circulation is thought to be in a state of near maximal vasodilation in healthy pregnancies. ${ }^{9}$ Changes in vasodilator tone may thus be important. ${ }^{13}$ Because the placenta is not innervated, vasomotor tone must be determined by endocrine and local mediators. Alongside nitric oxide and carbon monoxide, hydrogen sulfide is the third gasotransmitter modulating physiological vascular tone. Hydrogen sulfide is produced in the cardiovascular system by cystathionine- $\gamma$-lyase (CSE), using cysteine as a substrate. It acts by hyperpolarizing and relaxing smooth muscle cells (SMCs) by opening $\mathrm{K}_{\mathrm{ATP}}$ channels. ${ }^{14}$ The vasorelaxant effect of hydrogen sulfide is independent of the activation of soluble guanylyl cyclase, unlike that of nitric oxide and carbon monoxide. ${ }^{14}$ Recent evidence indicates a mutually dependent relationship between nitric oxide and hydrogen sulfide in the regulation of angiogenesis and endothelium-dependent vasorelaxation. ${ }^{15}$ CSE-null mice $\left(C t h^{-l-}\right)$ have markedly reduced serum hydrogen sulfide levels, which results in age-dependent development of hypertension, direct evidence that endogenous hydrogen sulfide can influence blood pressure. ${ }^{16}$ We recently reported the first evidence of the expression of CSE in the SMCs of the SVAs, and demonstrated $\mathrm{K}_{\mathrm{ATP}}$-dependent reduction of placental vascular resistance on exogenous administration of a hydrogen sulfide donor. ${ }^{17}$ Crucially, in cases of IUGR, as well as in preeclampsia accompanied by abnormal umbilical artery Doppler profiles, we found reduced expression of CSE in the smooth muscle cells of the stem villus arteries. This could be recapitulated by subjecting placental explants to hypoxia-reoxygenation (HR), a powerful inducer of oxidative stress. ${ }^{17}$ Other studies also confirmed reduced placental $\operatorname{CSE}^{18,19}$ and cystathionine $\beta$-synthase ${ }^{19,20}$ expression, and reduced plasma hydrogen sulfide levels in preeclamptic women. ${ }^{18}$ Administration of a CSE inhibitor to pregnant mice induced hypertension and liver damage, and promoted abnormal labyrinth vascularization in the placenta. ${ }^{18}$ These adverse effects could be reversed with administration of a slow-releasing hydrogen sulfidegenerating compound, GYY4137. ${ }^{18}$ Reduced bioavailability of hydrogen sulfide may thus be implicated in placental vasoconstriction, and a decrease in CSE/hydrogen sulfide activity may contribute to the pathogenesis of preeclampsia and intrauterine growth restriction.

In terms of passive tension, reduced distensibility of the resistance arteries can result in increased placental vascular resistance. No histological abnormalities in chorionic arteries in IUGR cases have been reported to date. In contrast, mounting evidence exists of functional changes in the SVAs in terms of differences of vessel morphology, including hypertrophy, hyperplasia, and luminal obliteration suggestive of vascular remodeling. ${ }^{21-23}$ These changes closely resemble those observed in other forms of vascular injury (eg, atherosclerosis and hypertension), ${ }^{24}$ which can be accounted for by SMC dedifferentiation.

The CSE/hydrogen sulfide pathway is essential for the maintenance of SMC phenotype, mediated by the upstream regulators miR-21 and specificity protein-1. $.^{25,26} \mathrm{miR}-21^{27,28}$ has been shown to be aberrantly overexpressed in some cardiovascular pathologies, such as cardiac hypertrophy ${ }^{29}$ or end-stage heart failure. ${ }^{30}$ We recently reported the involvement of miR-21 in down-regulation of the smooth muscle cell expression of CSE in human placentas, complicated by IUGR and with abnormal umbilical artery Doppler waveforms. ${ }^{17}$ Medial SMCs normally remain quiescent in a differentiated state. ${ }^{31}$ Differentiated SMCs express SMC-specific genes, such as myosin heavy chain (MHC), $\alpha$-smooth muscle actin (SMA), desmin, and calponin, and demonstrate appropriate contractility to contractile signals. Under stress conditions, SMCs dedifferentiate, adopting a synthetic phenotype characterized by increased proliferation, enhanced production of collagens and matrix metalloproteinases, and reduced expression of SMC-specific contractile markers. ${ }^{31-33}$ Aberrant proliferation and migration of SMCs are the underlying cause of many pathological vascular diseases, such as atherosclerosis.

Therefore, we hypothesized that pathological pregnancies are characterized by SMC dedifferentiation and SVA remodeling, and that this is because of oxidative stress secondary to poor placentation. This was tested by examining SMC marker expression in a well-characterized cohort of IUGR placental samples, and seeking to recapitulate vascular remodeling in vitro by subjecting SVA explants to HR. We also hypothesized that reduced hydrogen sulfide signaling is an important mechanism by which oxidative stress leads to SVA remodeling. We investigated this by correlating SMC marker expression with CSE in growthrestricted placental samples, and then showing in vitro that the changes produced by HR could be rescued by treatment with a hydrogen sulfide donor, and recapitulated under normoxia by inhibiting CSE. 


\section{Materials and Methods}

\section{Placenta Collection and Ethical Approval}

Normal-term placentas used for stem villus artery culture were collected immediately after delivery by elective cesarean section. Collection was done with approval from the Cambridge Local Ethics Committee, and with informed written patient consent. All pregnancies were uncomplicated singleton pregnancies at term (39 weeks). There were no known existing medical conditions. The most common indication for cesarean section was a history of previous section. The placenta was transported to the laboratory on ice.

Pathological placentas and respective term and preterm controls were collected with ethical approval from the Mount Sinai Hospital (Toronto, ON, Canada), after cesarean delivery. IUGR was defined according to established criteria ${ }^{34}$ (ie, fetal biometry was lower than the $10^{\text {th }}$ centile for gestational age, according to local reference, and umbilical artery Doppler flow velocity was abnormal). The cases included in this study were severe; they all presented with absent (Doppler score class II) or reversed (Doppler score class III) end-diastolic flow, which explains the early indications for delivery. The preterm control placentas were from women who had preterm but otherwise uneventful pregnancies, or late terminations of pregnancy for medical reasons. Fetal growth had been normal and birth weight was always higher than the $10^{\text {th }}$ percentile. The umbilical and uterine Doppler measurements were normal. The membranes were never ruptured for $>12$ hours and signs of chorioamnionitis were also excluded by histological studies. The cases included in this study were composed of four late terminations of pregnancy (because of autosomal recessive polycystic kidneys or renal agenesis), three cases of early premature rupture of membranes (no chorioamnionitis) and one case of early preterm labor. It is essential to include gestationally matched preterm controls. However, preterm deliveries by definition are not normal controls. We therefore included an additional group of term controls from healthy uneventful pregnancies with normal umbilical and uterine artery Doppler waveforms. Placental tissue was washed and fixed in $4 \%$ paraformaldehyde and processed for immunohistochemistry, or frozen immediately and stored at $-80^{\circ} \mathrm{C}$.

\section{Placenta Dissection and Culture of SVA Explants}

The placenta was dissected on ice in a glove box equilibrated at $10 \% \mathrm{O}_{2} / 85 \% \mathrm{~N}_{2} / 5 \% \mathrm{CO}_{2}$. For dissection of SVAs, the placenta was bisected through the region of umbilical cord insertion, the chorionic plate was then reflected, and the underlying tissue was teased away until an SVA was identified. The SVAs were further exposed by removing their trophoblastic covering, dissected proximally to their origin from the chorionic arteries, and transected. The SVAs were also dissected distally, and transected after they had branched two to three times, as beyond that the vessels were too small for effective removal.

SVA explants were cultured in Dulbecco's modified Eagle's medium/F-12 medium ( $10 \%$ fetal calf serum) at $37^{\circ} \mathrm{C}$ for 1 or 3 days. Normoxic controls were maintained constantly at $10 \% \mathrm{O}_{2} / 85 \% \mathrm{~N}_{2} / 5 \% \mathrm{CO}_{2}$. Explants that were subjected to HR were incubated in repeated 12-hour cycles of 6 hours in $1 \% \mathrm{O}_{2} / 94 \% \mathrm{~N}_{2} / 5 \% \mathrm{CO}_{2}$, followed by 6 hours in normoxic conditions (Xvivo System G300C; BioSpherix, Parish, NY). All SVAs were flash frozen in liquid nitrogen and then stored at $-80^{\circ} \mathrm{C}$, for protein analysis and measurements of hydrogen sulfide production. Where CSE inhibition was required, DL-propargylglycine (Sigma, Pool, UK) was added to a final concentration of $10 \mathrm{mmol} / \mathrm{L}$. Diallyl trisulfide (DATS; LKT Laboratories Inc., St Paul, MN) was used at $500 \mu \mathrm{mol} / \mathrm{L}$.

\section{Measurement of Endogenous Hydrogen Sulfide Production}

The protocol used was modified from Zhao et al. ${ }^{14}$ SVA lysate $(80 \mu \mathrm{L})$ was incubated with $2 \mathrm{mmol} / \mathrm{L}$ L-cysteine, $2 \mathrm{mmol} / \mathrm{L}$ pyridoxal $3^{\prime}$-phosphate (Sigma), and a $100 \mathrm{mmol} / \mathrm{L}$ $\mathrm{KH}_{2} \mathrm{PO}_{4}$ buffer in a 1.5 -mL Eppendorf tube sealed with Parafilm, with a total reaction volume of $1 \mathrm{~mL}$. The reaction proceeded at $37^{\circ} \mathrm{C}$ for 1 hour, before being stopped on ice. Then, $10 \% \mathrm{ZnAc}$ (Alfar Aesar, Lancashire, UK; $500 \mu \mathrm{L}$ ) was injected into the Eppendorf tube, before immediately sealing the hole with Parafilm. This amount of $\mathrm{Zn}^{2+}$ was in large molar excess of the hydrogen sulfide that was expected to be produced, because $\mathrm{Zn}_{3}\left(\mathrm{PO}_{4}\right)_{2}$ is also sparingly soluble and would be precipitated out. The precipitate was collected by centrifugation at $21,130 \times g$ at $0^{\circ} \mathrm{C}$ for 10 minutes, and resuspended in a final volume of $1 \mathrm{~mL}$, with $2.4 \mathrm{mmol} / \mathrm{L}$ $\mathrm{N}, \mathrm{N}$-dimethyl-p-phenylenediamine (Sigma), $4.5 \mathrm{mmol} / \mathrm{L}$ $\mathrm{FeCl}_{3}$ (Alfa Aesar), $\mathrm{pH}-0.018$. Absorbance at $670 \mathrm{~nm}\left(\mathrm{~A}_{670}\right)$ was measured after 20 minutes. For every experiment, an internal control reaction with ZnAc omitted was performed, for subtraction to obtain the hydrogen sulfide-specific signal.

\section{Immunohistochemical Staining and Quantification}

Immunohistochemistry was performed as previously described. ${ }^{35}$ Briefly, paraformaldehyde-fixed tissues were dehydrated and embedded in paraffin wax. Sections $(7 \mu \mathrm{m}$ thick) were dewaxed, rehydrated, and incubated in $3 \% \mathrm{H}_{2} \mathrm{O}_{2}$ for 15 minutes to block endogenous peroxidase activity, followed by 1 hour in nonimmune goat serum to prevent nonspecific antibody binding. Sections were then incubated overnight at $4^{\circ} \mathrm{C}$ with the appropriate primary antibodies antiCSE (Proteintech, Cambridge, UK; 12217-1-AP), anti-MHC (Abcam, Cambridge, UK; ab82255), anti-SMA (Dako, Ely, UK; M0851), anti-cellular retinol-binding protein 1 (CRBP1; Abcam; ab119056), anti-desmin (Abcam; ab32362), or anti-matrix metalloproteinase (MMP) 2 (Abcam; ab86607), which were then detected with avidinconjugated secondary antibodies, and visualized with a 
Vectastain Elite ABC kit (Vector Laboratories, Peterborough, UK) and SigmaFast DAB (Sigma). Sections were then lightly counterstained with hematoxylin, before being dehydrated and mounted. Where antigen retrieval was required, it was performed after the hydrogen peroxide incubation in a pressure cooker for 2 minutes in $1 \mathrm{mmol} / \mathrm{L}$ Tris $-0.1 \mathrm{mmol} / \mathrm{L}$ EDTA buffer, pH 9.0.

Stained slides were scanned using a NanoZoomer scanner (Hamamatsu, Welwyn Garden City, UK). Each slide was visualized, and several images of stem villus arteries (6 to 10 per placenta) were captured, with the observer (T.C.-D.) blind to the study group. The staining intensity of the markers of SMC differentiation in all vessels was analyzed semiquantitatively, using the score of 0 to 2 ( 0 indicates negative; 1 , weak; and 2 , strong) by two independent observers (T.C.-D. and L.L.). The scores were averaged for each slide, and interindividual variation was assessed by computing the Pearson correlation coefficient, $R$. $R$ was approximately 0.9 for the markers examined.

\section{Morphometric Analysis of Vessel Wall Thickness and Lumen}

Placental sections were stained with hematoxylin and eosin and scanned as described in the previous paragraph. Vessel and lumen diameter were measured using ImageJ software version 1.46r (NIH, Bethesda, MD; http://imagej.nih.gov/ij). After calibrating the software, the vessel diameter $\left(D_{\mathrm{V}}\right)$ and lumen diameter $\left(D_{L}\right)$ were measured four times for each SVA by drawing lines across the vessel or lumen cross section, and the mean values recorded. The wall thickness was calculated as follows: $\left(D_{V}-D_{L}\right) / 2$.

The ratio of vessel wall thickness and wall lumen diameter was calculated for each vessel. Measurements from at least six stem villus arteries were taken per placenta. A mean value of the measured variables was calculated for each placenta, and a mean of means \pm SD was obtained for each placental group.

\section{RNA Isolation and Quantitative Real-Time RT-PCR Analysis}

Total RNA was isolated from snap-frozen placental tissue using an RNAeasy kit (Qiagen, Crawley, UK). RNA was quantified by spectrophotometry (Nanodrop Technologies, Wilmington, DE) and integrity assessed using an Agilent 2100 bioanalyzer (Agilent Technologies UK Limited, Craven Arms, UK). In brief, $20 \mu \mathrm{g}$ of total RNA from each sample was reverse transcribed using a master mix containing SuperScript II Reverse Transcriptase in the First Strand Buffer with $0.1 \mathrm{~mol} / \mathrm{L}$ dithiothreitol (Invitrogen, Paisley, UK) and $50 \mathrm{ng} / \mathrm{mL}$ random hexamers (Sigma). The DNA Engine Opticon 2 Sequence Detection System (BioRad Laboratories, Watford, UK) was used to perform realtime PCR according to the manufacturer's protocols (using TaqMan FAM1 dye). Ct values for each transcript were compared with those for 18S rRNA (dCt obtained), and these values were compared to term control samples (ddCt values are reported). All primers and probes were obtained from Applied Biosystems (ABI, Warrington, UK).

\section{Western Blots}

Frozen SVA explant samples were homogenized in ice-cold lysis buffer (1 mL of buffer per $100 \mathrm{mg}$ tissue) containing 20 $\mathrm{mmol} / \mathrm{L}$ Tris, $\mathrm{pH} 7.4,1 \mathrm{mmol} / \mathrm{L}$ EGTA, $0.01 \%$ Triton X-100, 1 $\mathrm{mmol} / \mathrm{L}$ sodium pyrophosphate, $1 \mathrm{mmol} / \mathrm{L}$ sodium orthovanadate, $10 \mathrm{mmol} / \mathrm{L} \beta$-glycerol phosphate, $50 \mathrm{mmol} / \mathrm{L}$ sodium fluoride, and a complete miniprotease inhibitor cocktail (Roche, Roche Diagnostics, East Sussex, UK). Tissue homogenates were centrifuged at $15,000 \times g, 4^{\circ} \mathrm{C}$ for 20 minutes. Protein concentrations were determined on the supernatant using a BCA protein assay kit (Sigma). Lysates were mixed with $3 \times$ SDS-PAGE sample buffer, boiled for 5 minutes, and allowed to return to room temperature. Equal amounts of protein (30 to $50 \mu \mathrm{g}$ ) were separated by SDS-PAGE, using $7.5 \%$ to $12.5 \%$ polyacrylamide resolving gels, and transferred onto nitrocellulose membrane (Invitrogen), and subjected to immunoblot analysis. Membranes were blocked for 1 hour at $25^{\circ} \mathrm{C}$ in $5 \%$ milk diluted in Tris-buffered saline and $0.1 \%$ Tween 20 and incubated with the following primary antibodies overnight at $4^{\circ} \mathrm{C}$ : anti-CSE (43 kDa; Proteintech; 122171-AP), anti-MHC (227 kDa; Abcam; ab82255), anti-SMA (42 kDa; Dako; M0851), anti-CRBP1 (16 kDa; Abcam; ab119056), anti-desmin (52 kDa; Abcam; ab32362), or antihydroxynonenal (multibands, a single strong band of approximately $30 \mathrm{kDa}$ quantified; Calbiochem, Watford, UK). After washing and incubating with secondary antibodies, immunoreactive proteins were visualized by the ECL plus chemiluminescence system, following the manufacturer's instructions (Amersham Biosciences, Little Chalfont, UK). Protein bands were quantified using ImageJ. Protein loading was normalized against $\beta$-actin staining. The values are expressed as a percentage of the control lysate (100\%) for each experiment.

\section{Statistical Analysis}

Data are expressed as means $\pm \mathrm{SD}$. Comparisons were made using a two-tailed $t$-test or analysis of variance with a Tukey's multiple comparison post hoc test where appropriate. Differences were considered to be significant at $P \leq 0.05$. Correlations were tested using Pearson's correlation coefficient at $P \leq 0.05$.

\section{Results}

\section{IUGR Is Associated with SVA Remodeling and SMC Dedifferentiation}

A well-characterized cohort of IUGR placentas $(n=34)$ and two control groups, term $(n=8)$ and preterm $(n=8)$, 
Table 1 Patient Details

\begin{tabular}{lllcll}
\hline Group & Patients, $n$ & Maternal age, years & Gestational age, weeks & Birth weight, g & Placental weight, $g$ \\
\hline TC & 8 & $33.3 \pm 4.2$ & $38.8 \pm 0.6$ & $3355 \pm 664$ & $462.8 \pm 77$ \\
PTC & 8 & $31.4 \pm 4.6$ & $29 \pm 3^{*}$ & $1289 \pm 427^{*}$ & $278.1 \pm 72.1^{*}$ \\
IUGR & 34 & $33.1 \pm 5.2$ & $29.1 \pm 2.7^{* \dagger}$ & $679 \pm 270^{* \dagger}$ & $157.2 \pm 67.8^{* \dagger}$ \\
\hline
\end{tabular}

Data are expressed as means \pm SD.

${ }^{*} P<0.05$ versus TC, using one-way analysis of variance, Tukey's multiple comparison test.

${ }^{\dagger} P<0.05$ versus PTC, using one-way analysis of variance, Tukey's multiple comparison test.

IUGR, intrauterine growth restriction; PTC, preterm control; TC, term control.

were included in this study (Table 1). There were no significant differences in maternal age among the three placental group studies. The gestational ages of the preterm control and IUGR groups were not different, whereas the term control group had a significantly longer gestational age by definition. The two IUGR groups had the lowest birth weights and placental weights, which were significantly different compared to both preterm and term control pregnancies (Table 1).
We found MHC to be markedly reduced at the protein level in placental samples from the IUGR group (Figure 1, A and B), and at the mRNA level compared with preterm controls (Figure 1C). In addition, there was a strong positive correlation between birth weight and MHC protein $(r=0.7992$, $P<0.0001$ ) (Figure 1D). Similarly, SMA was significantly reduced in the IUGR placentas (Figure 1, E and F), and there was a reduction in actin (ACTA) mRNA level between the IUGR and preterm control samples (Figure $1 \mathrm{G}$ ). There was a
A

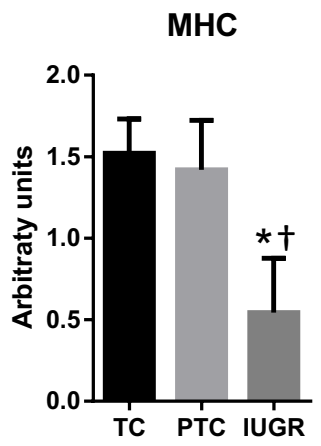

$\mathbf{E}$

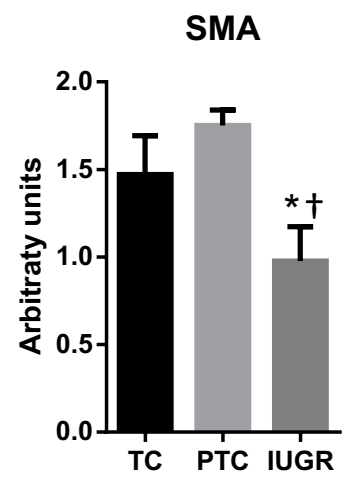

B

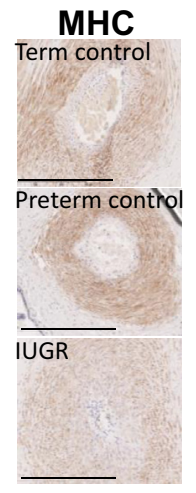

$\mathbf{F}$

SMA

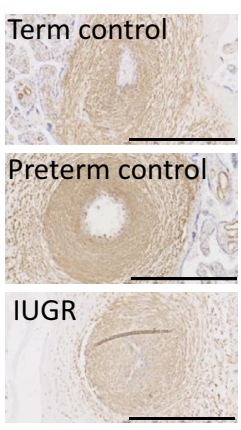

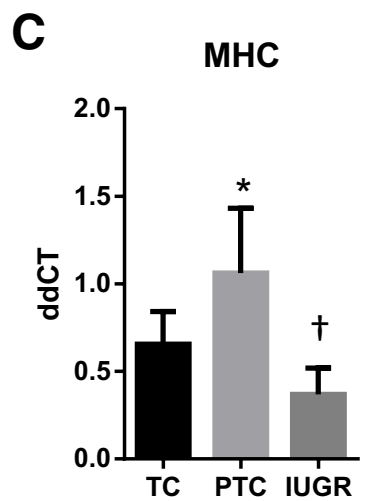

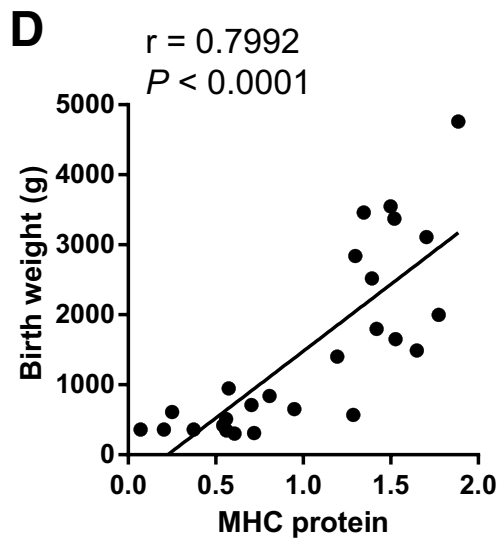

H

ACTA

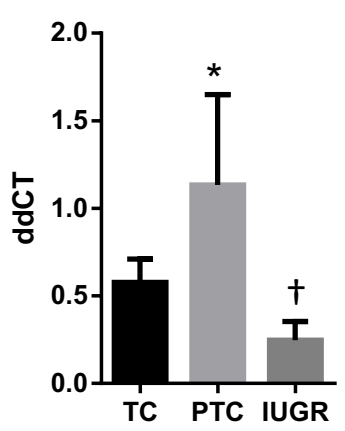

$$
r=0.5012
$$$$
P=0.0025
$$

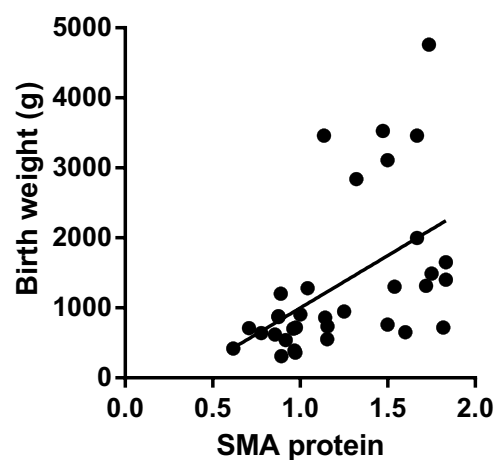

Figure 1 Quantification of myosin heavy chain (MHC; A-D) and $\alpha$-smooth muscle actin (SMA; $\mathbf{E}-\mathbf{H})$ protein in placental stem villus arteries (SVAs) and mRNA expression in whole placental homogenates of term controls (TC) and preterm controls (PTC) versus intrauterine growth restriction (IUGR) placentas. Human placental sections were immunostained with anti-MHC (A and $\mathbf{B}$ ) or anti-SMA (E and $\mathbf{F}$ ), scanned, and the staining within SVAs quantified using ImageJ $(\mathbf{A}$ and $\mathbf{E})$. Representative images are shown in $\mathbf{B}$ and $\mathbf{F}$. mRNA was extracted from whole placental homogenates and the mRNA expression of MHC (C) and ACTA (SMA; G) quantified using TaqMan. The protein level of MHC (D) and SMA (H) correlate with birth weight. Data are expressed as means \pm SD (A, C, E, and $\mathbf{G})$. $n=6(\mathbf{A}$ and $\mathbf{B}$, TC and PTC, and $\mathbf{E}$ and $\mathbf{F}$, TC); $n=13$ (A and B, IUGR); $n=7$ (E and $\mathbf{F}$, PTC); $n=18$ (E and $\mathbf{F}$, IUGR); $n=5$ per group (C and $\mathbf{~})$. ${ }^{*} P<0.05$ versus TC, using one-way analysis of variance, Tukey's multiple comparison test; ${ }^{\dagger} P<0.05$ versus PTC, using one-way analysis of variance, Tukey's multiple comparison test. Scale bars $=400 \mu \mathrm{m}(\mathbf{B}$ and $\mathbf{F})$. 
significant correlation between birth weight and SMA protein $(r=0.5012, P=0.0025)$ (Figure $1 \mathrm{H})$. The protein level and mRNA expression of desmin, a marker of mature SMC, were significantly reduced in the stem villi of the IUGR placentas (Figure 2, A-C), and there was a significant correlation between birth weight and desmin protein expression $(r=0.6754$, $P=0.0006$ ) (Figure 2D). In contrast, the protein levels of the dedifferentiation markers CRBP1 (Figure 2, E-H) and MMP2 (Figure 3, A-C) were significantly increased in the SVAs of IUGR placentas, and there were negative correlations between birth weight and CRBP1 protein levels $(r=-0.4596$, $P=0.0071$ ) (Figure $2 \mathrm{H}$ ), and MMP2 protein levels $(r=-0.5729, P=0.0083$ ) (Figure 3C).

In terms of morphometric parameters, there was no difference in the vessel wall thickness among the groups (Figure 4A). However, the lumen diameter was significantly reduced in the IUGR placentas (Figure 4B) compared to both term and preterm controls, and the wall thickness/ lumen ratio was thus significantly increased (Figure 4C).
IUGR Is Associated with Low Protein and mRNA Expression of CSE in Human SVAs

Quantification of the CSE expression revealed a significant reduction in the immunostaining of CSE in the IUGR group (Figure 3, D and E), and a significant reduction of CSE mRNA in whole placental homogenates of IUGR samples, compared with age-matched preterm controls (Figure 3F). There was a positive correlation between birth weight and CSE expression $(r=0.6580$, $P<0.0001$ ) (Figure $3 \mathrm{G}$ ). In addition, the protein and mRNA levels of CSE showed positive correlations with the protein level and mRNA expressions of the contractile protein markers MHC, SMA, and desmin: CSE versus MHC protein: $r=0.8599, P \leq 0.0001$ (Figure 4D); CSE versus MHC mRNA: $r=0.6699, P<0.0001$ (Figure 4E); CSE versus SMA protein: $r=0.8269$, $P<0.0001$ (Figure 4F); CSE versus SMA (ACTA) mRNA: $r=0.4804, P=0.0255$ (Figure 4G); CSE
A

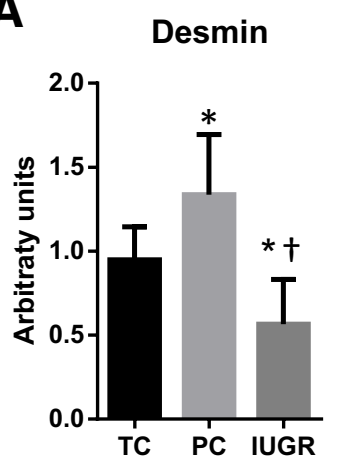

E

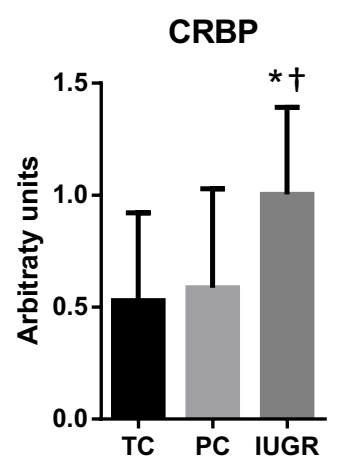

B

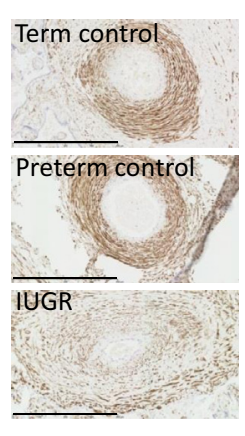

$\mathbf{F}$

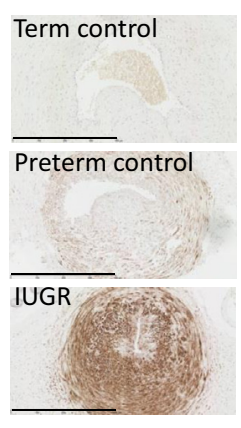

C

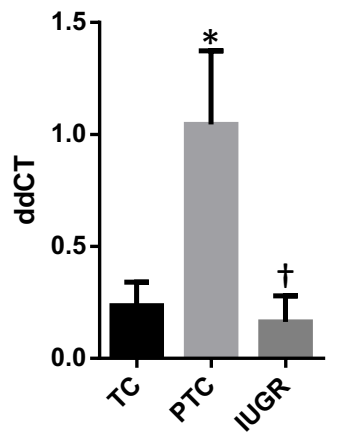

G

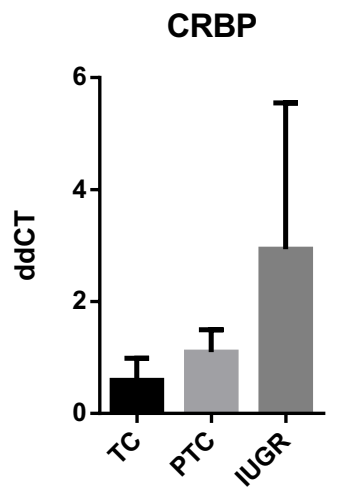

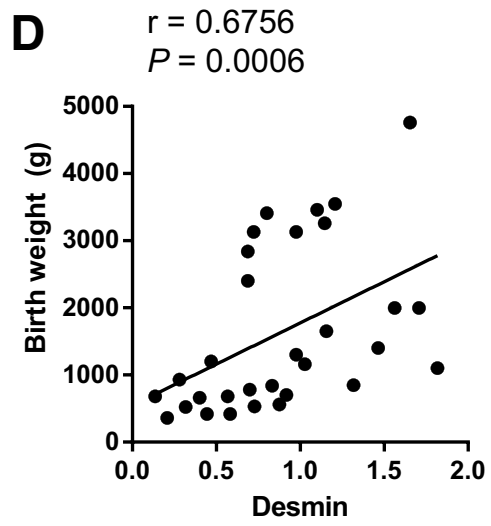

H

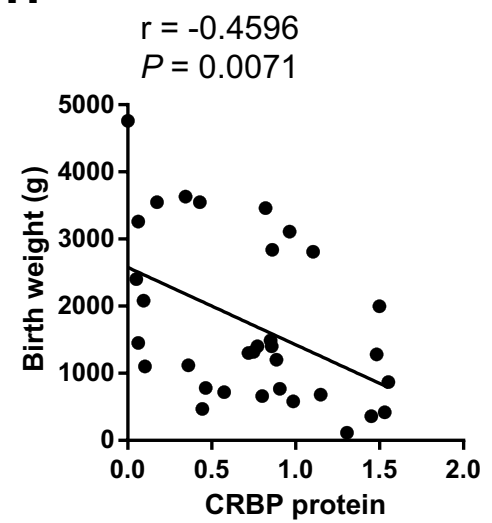

Figure 2 Quantification of desmin (A-D) and CRBP1 (E-H) protein in placental stem villus arteries (SVAs) and mRNA expression in whole placental homogenates of term (TC) and preterm (PTC) controls versus intrauterine growth restriction (IUGR) placentas. Human placental sections were immunostained with anti-desmin (A and B) or anti-CRBP1 (E and F), scanned, and the staining within SVAs quantified using ImageJ (A and E). Representative images are shown in $\mathbf{B}$ and $\mathbf{F}$. mRNA was extracted from whole placental homogenates and the mRNA expression of desmin (C) and CRBP (G) quantified using TaqMan. The protein level of desmin (D) and CRBP (H) was correlated with birth weight. Data are expressed as means \pm SD (A, C, E, and $\mathbf{~}) . n=8$ (A and $\mathbf{B}$, TC); $n=7$ (A and $\mathbf{B}$, PTC, and $\mathbf{E}$ and $\mathbf{F}$, TC and PTC); $n=16$ (A and B, IUGR); $n=18$ (E and $\mathbf{F}$, IUGR); $n=5$ per group ( $\mathbf{C}$ and $\mathbf{G}) .{ }^{*} P<0.05$ versus TC, using one-way analysis of variance, Tukey's multiple comparison test; ${ }^{\dagger} P<0.05$ versus $\mathrm{PTC}$, using one-way analysis of variance, Tukey's multiple comparison test. Scale bars $=400 \mu \mathrm{m}$ (B and F). 
A

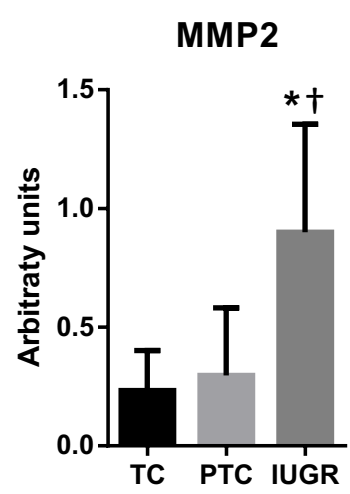

D

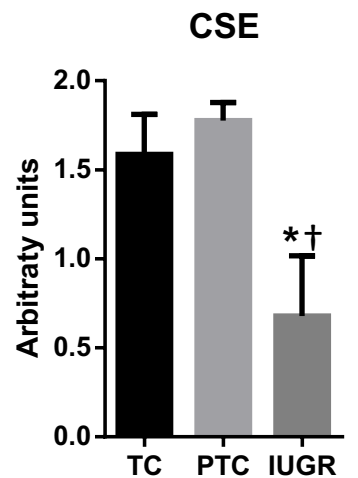

B

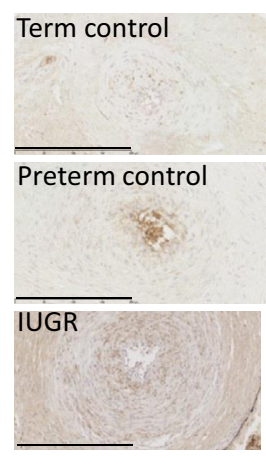

E

CSE

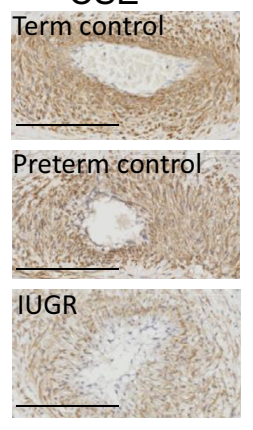

C

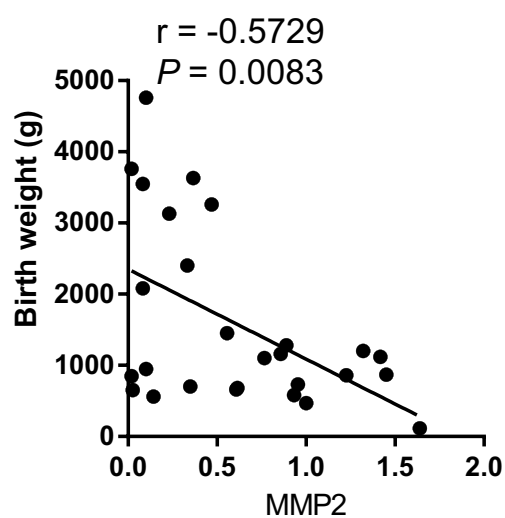

F

G

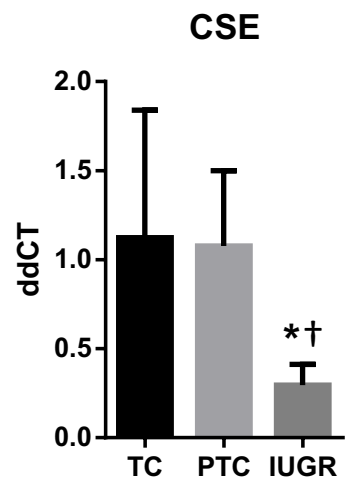

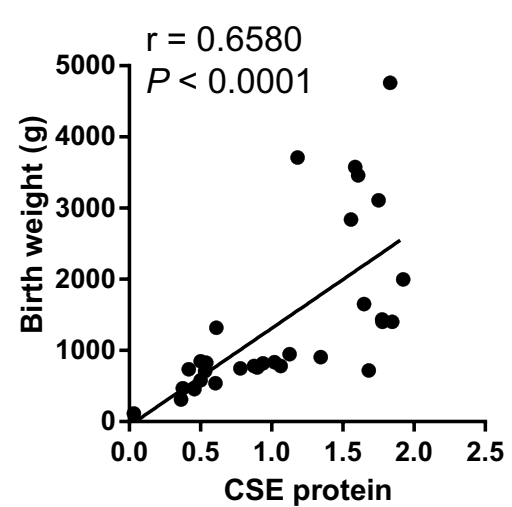

Figure 3 Quantification of matrix metalloproteinase (MMP) 2 (A-C) and cystathionine- $\gamma$-lyase (CSE; D-G) protein in placental stem villus arteries (SVAs) and mRNA expression in whole placental homogenates of term (TC) and preterm (PTC) controls versus intrauterine growth restriction (IUGR) placentas. Human placental sections were immunostained with anti-MMP2 (A and B) or anti-CSE (E and F), scanned, and the staining within SVAs quantified using ImageJ (A and E). Representative images are shown in B and E. F: mRNA was extracted from whole placental homogenates and the mRNA expression of CSE quantified using TaqMan. The protein expression of MMP2 (C) and CSE (G) correlates with birth weight. Data are expressed as means \pm SD $(\mathbf{A}, \mathbf{D}$, and $\mathbf{F}) . n=6(\mathbf{A}, \mathbf{B}, \mathbf{E}$, and $\mathbf{F}$, TC and PTC); $n=15$ (A and B, IUGR); $n=20$ (E and $\mathbf{F}$, IUGR); $n=5$ per group (F). * $P<0.05$ versus TC, using one-way analysis of variance, Tukey's multiple comparison test; ${ }^{\dagger} P<0.05$ versus PTC, using one-way analysis of variance, Tukey's multiple comparison test. Scale bars $=200 \mu \mathrm{m}(\mathbf{B}$ and $\mathbf{E})$.

versus desmin protein: $r=0.6518, P<0.0018$ (Figure 4H).

Oxidative Stress Results in Smooth Muscle Dedifferentiation of Stem Villus Artery Explants in Vitro

We attempted to recapitulate the changes seen in the vascular tree of IUGR placentas by subjecting stem villus artery explants dissected from healthy term placentas to oxidative stress induced by HR in vitro for 3 days. HR induced oxidative stress, as indicated by increased expression of hydroxynonenal (Figure 5A). In addition, the HR challenge had a profound effect on reducing the markers of SMC differentiation, MHC and SMA, and desmin, and increasing the dedifferentiation marker CRBP1, compared to normoxic controls (Figure 5A). These changes are indicative of oxidative stress-mediated vascular remodeling on exposure to $\mathrm{HR}$.
Hypoxia-Reoxygenation Reduces the CSE Expression and Hydrogen Sulfide Production in Stem Villus Artery Explants in Vitro

In addition to inducing oxidative stress and vascular remodeling, the HR challenge had a profound effect on reducing the CSE enzyme, after 3 days' exposure (Figure 5B). These changes in the CSE enzyme expression were functional as they were associated with a significant decrease in hydrogen sulfide gas production in HR-treated explants compared to normoxic controls (Figure 5C).

CSE Inhibition Recapitulates, and Addition of a Natural Hydrogen Sulfide Donor DATS Prevents, the Effect of HR on SMC Dedifferentiation

Given the role of CSE in vascular remodeling ${ }^{25,26}$ and the evidence linking vascular remodeling with increased 
A

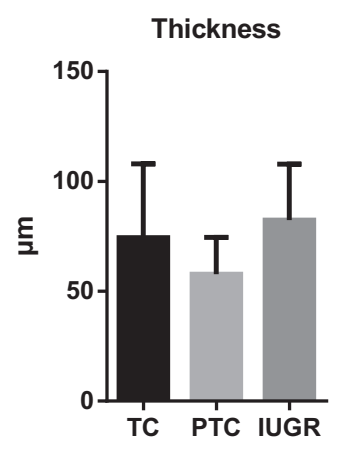

E

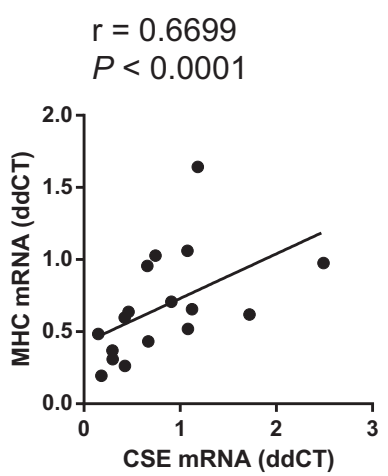

B

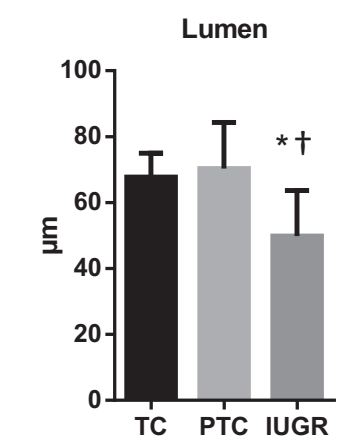

$\mathbf{F}$

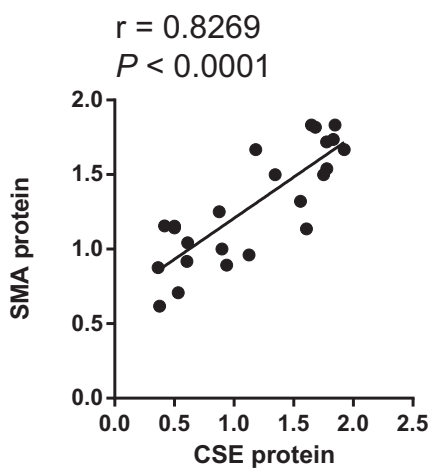

C

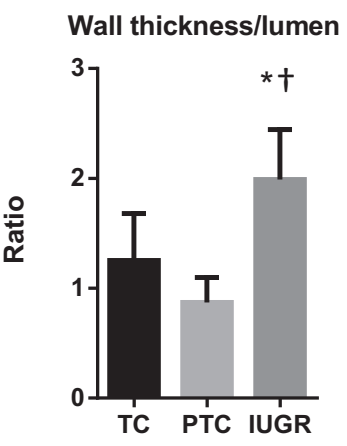

G

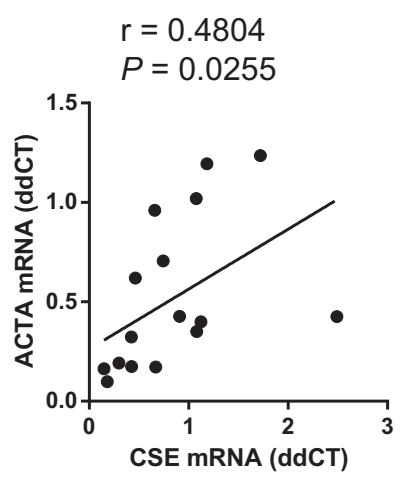

D

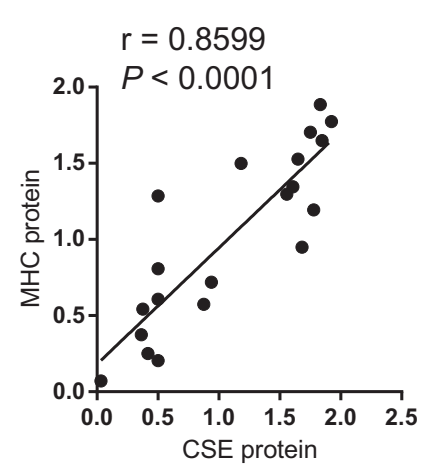

H

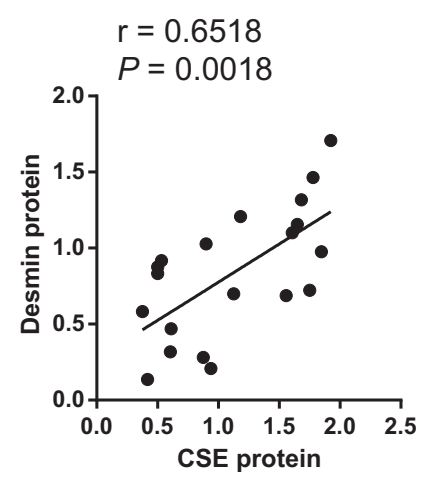

Figure 4 Morphometric analysis of the stem villus arteries and correlations of smooth muscle markers against cystathionine- $\gamma$-lyase (CSE) expression. A-C: Human placental sections were stained with hematoxylin and eosin and scanned using a NanoZoomer. Vessel thickness and lumen diameter were measured using ImageJ and the thickness/lumen ratio expressed. Measurements from at least six stem villus arteries were taken per placenta. D-H: A mean value of the measured variables was calculated for each placenta, and a mean of means \pm SD obtained for each placental group. Correlations were tested between protein level and mRNA expression of CSE and the contractile markers myosin heavy chain (MHC; $\mathbf{D}$ and $\mathbf{E}), \alpha$-smooth muscle actin (SMA; F), ACTA (G), and desmin $(\mathbf{H})$. Data are expressed as means \pm SD $(\mathbf{A}-\mathbf{C}) . n=6[\mathbf{A}-\mathbf{C}$, term control (TC)], $n=7[\mathbf{A}-\mathbf{C}$, preterm control (PTC)]; $n=16[\mathbf{A}-\mathbf{C}$, intrauterine growth restriction (IUGR)]. ${ }^{*} P<0.05$ versus TC, using one-way analysis of variance, Tukey's multiple comparison test; ${ }^{\dagger} P<0.05$ versus $\mathrm{PTC}$, using one-way analysis of variance, Tukey's multiple comparison test.

resistance, we tested the effect of CSE inhibition (using DL-propargylglycine) on vascular remodeling of SVAs in vitro. Treatment of SVA explants with the CSE inhibitor DL-propargylglycine under normoxic conditions resulted in increased oxidative stress, as demonstrated by increased hydroxynonenal immunostaining (Figure 5D). It also led to a significant reduction of MHC, SMA, and desmin protein, and an increase in the level of CRBP1, thus indicating SMC dedifferentiation (Figure 5D). These results confirm that CSE inhibition is sufficient to recapitulate the SMC remodeling effects on SVAs.

We tested whether these processes could be prevented by treating placental explants with a natural donor, DATS. We challenged placental stem villus explants with HR or the CSE inhibitor DL-propargylglycine (cultured under normoxia) for 3 days. Both stimuli reduced the differentiation markers MHC, SMA, and desmin, and increased CRBP1 and the oxidative stress marker hydroxynonenal, as before (Figure 6). More important, addition of DATS prevented this dedifferentiated phenotype by maintaining the levels of MHC, SMA, desmin, and CRBP1 (Figure 6), confirming the important role of the CSE/hydrogen sulfide pathway in maintaining the differentiated SMC phenotype.

\section{Discussion}

We provide the first molecular evidence that the increased umbilical artery resistance seen in IUGR pregnancies is associated with remodeling of stem villus arteries, characterized by pathological smooth muscle dedifferentiation. Compared to term or preterm controls, SVAs of IUGR placentas contain reduced levels of the contractile proteins MHC, SMA, and desmin, and increased levels of the markers of dedifferentiation CRBP1 and MMP2. These changes are associated with an increase in the wall thickness/lumen ratio, and a reduction in vessel lumen diameter. The protein levels of MHC, SMA, desmin, CRBP, and MMP2 strongly positively correlated with birth weight. CSE 
A
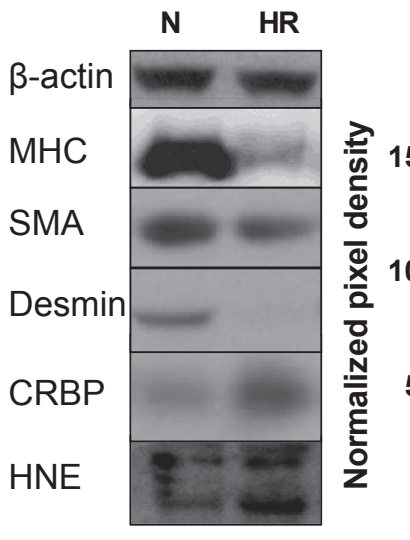

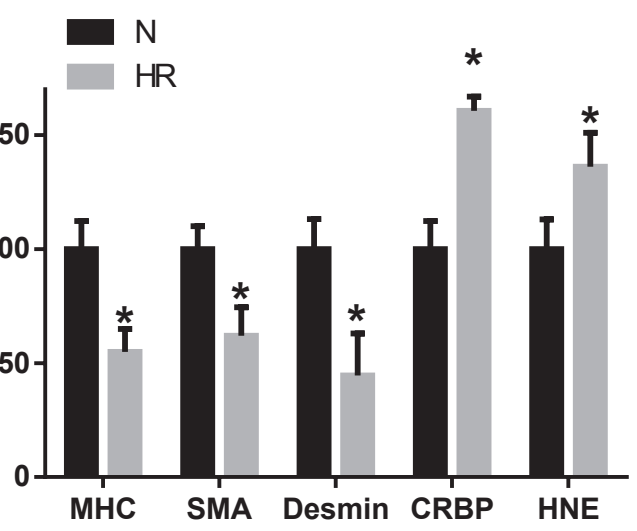

B
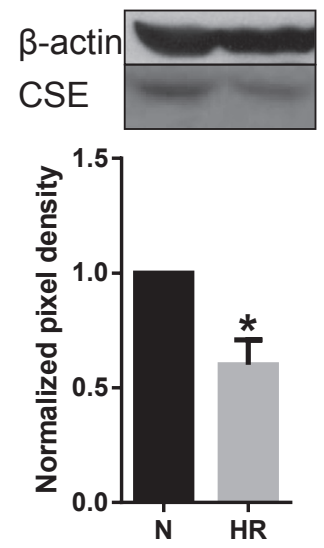

C

Hydrogen sulfide production

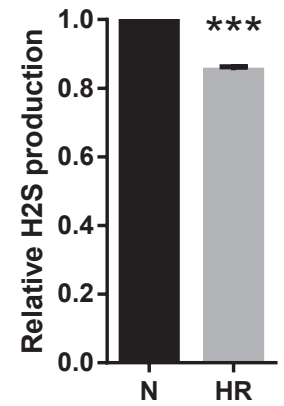

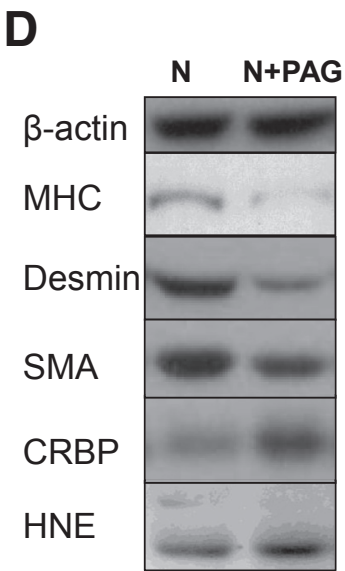

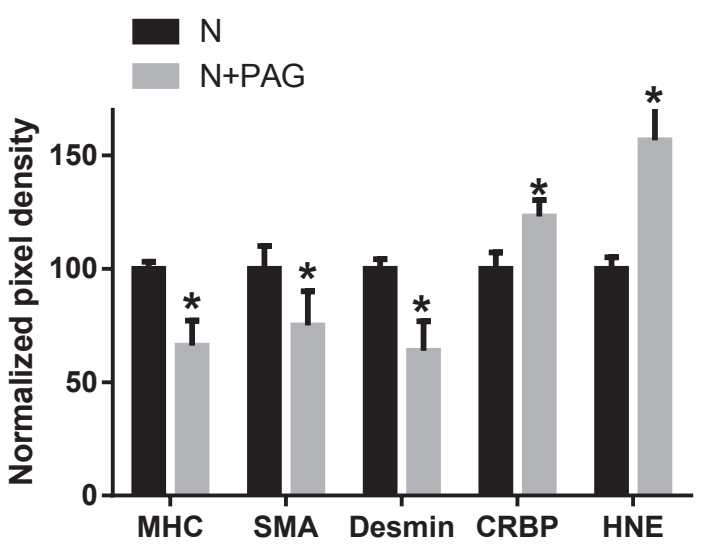

Figure 5 Effect of chronic oxidative stress $(\mathbf{A}-\mathbf{C})$ or cystathionine- $\gamma$-lyase (CSE) inhibition (D) on smooth muscle cell (SMC) markers (A) as a functional index of SMC synthetic phenotype, and on CSE expression and hydrogen sulfide production (B and C). A: Levels of SMC markers in isolated stem villus arteries (SVAs) were quantified by Western blotting. A representative Western blot is shown on the left panel, and the average profile of the markers on the right panel. Hydroxynonenal (HNE) was also probed to confirm that hypoxia-reoxygenation (HR) increased oxidative stress. B: Western blot analysis of CSE in isolated SVAs subject to chronic (3 days) oxidative stress. A representative Western blot is shown on the top panel, and the average profile shown on the bottom panel. C: In vitro hydrogen sulfide production capacity of isolated SVAs. Equal amounts of protein from SVAs cultured in normoxia (N) or HR were used. D: SMC markers in isolated SVAs treated with $1 \mathrm{mmol} / \mathrm{L}$ CSE inhibitor DL-propargylglycine (PAG) under normoxia $\left(10 \% \mathrm{O}_{2}\right)$ for 3 days were quantified by Western blotting. A representative Western blot is shown on the left panel, and the average profile of the markers on the right panel. HNE was also probed to test whether PAG treatment increased oxidative stress. $n=4$ (A-D); $n=3(\mathbf{B}) .{ }^{*} P<0.05,{ }^{*} * *<0.001$ versus N. MHC, myosin heavy chain; SMA, $\alpha-$ smooth muscle actin.

protein and mRNA were also reduced, and correlated strongly with the extent of SMC dedifferentiation at both protein and mRNA levels. The physiological and clinical importance of hydrogen sulfide signaling was further underlined by the fact that CSE levels explained $43 \%$ of the variation in birth weight, and 50\% of the variation among growth-restricted pregnancies. Mechanistically, we could recapitulate the vascular remodeling by subjecting placental SVA explants to hypoxia-reoxygenation, which led to reduced hydrogen sulfide production, or by inhibiting the activity of the CSE protein. The differentiated smooth muscle phenotype could be rescued by concomitant treatment with the hydrogen sulfide donor DATS. Collectively, these data suggest the following: i) stem villi from IUGR placentas show evidence of vascular remodeling, ii) oxidative stress is able to induce SMC dedifferentiation and SVA remodeling, and iii) a reduction in hydrogen sulfide signaling is necessary and sufficient for oxidative stress to induce SVA remodeling.

Although there is no reduction in the number of vessels per stem villus in IUGR, ${ }^{36}$ differences exist in the vessel morphology. ${ }^{21-23}$ Fok et $\mathrm{al}^{21}$ reported hypertrophy of the intima and media of SVAs, and correlated these findings with the degree of Doppler cord flow abnormality. Salafia et $\mathrm{al}^{22}$ reported significantly more fetal stem vessels with medial hyperplasia and luminal obliteration in cases of IUGR with absent end-diastolic flow; lesions suggestive of vascular remodeling and/or damage by pathological 
A

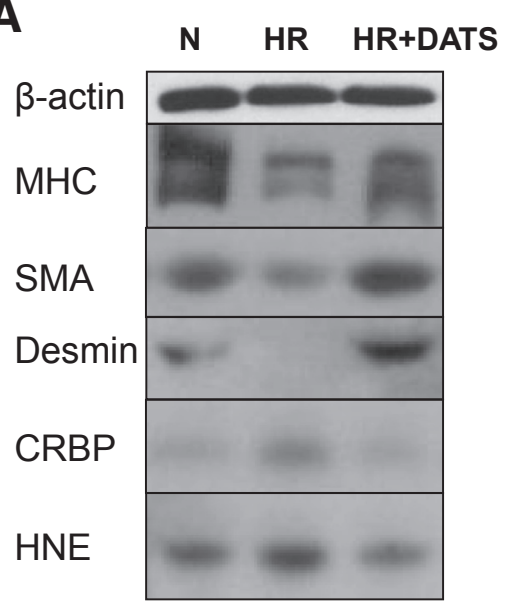

B

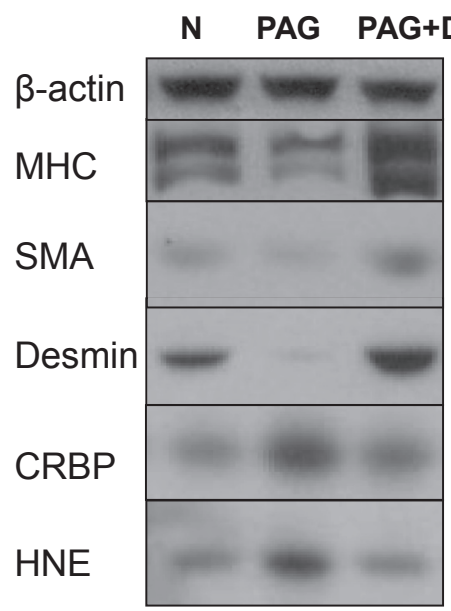

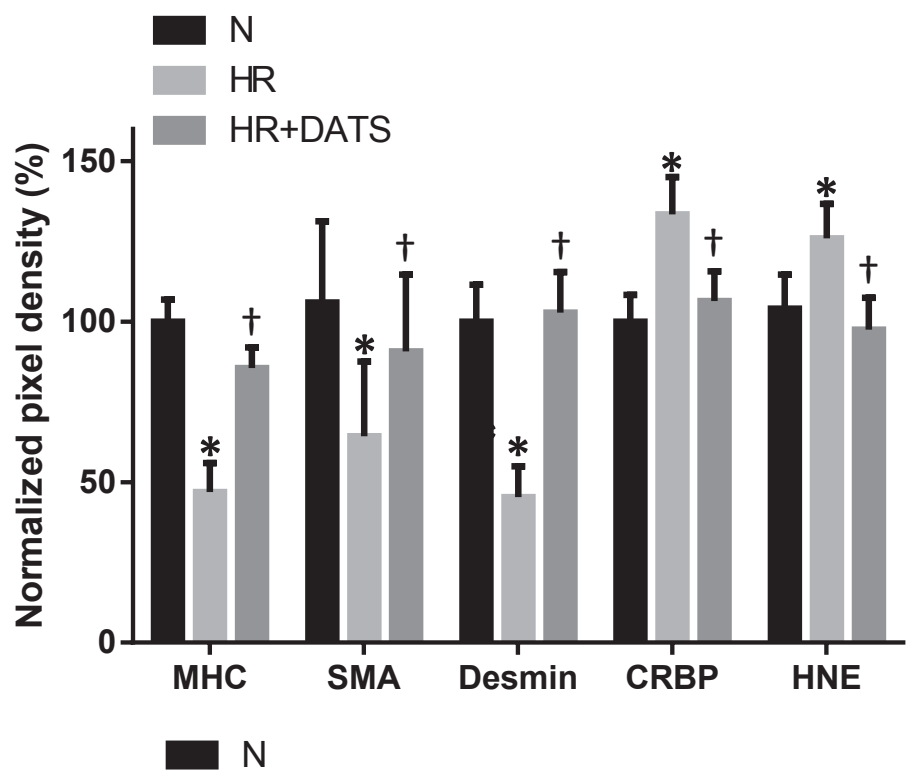

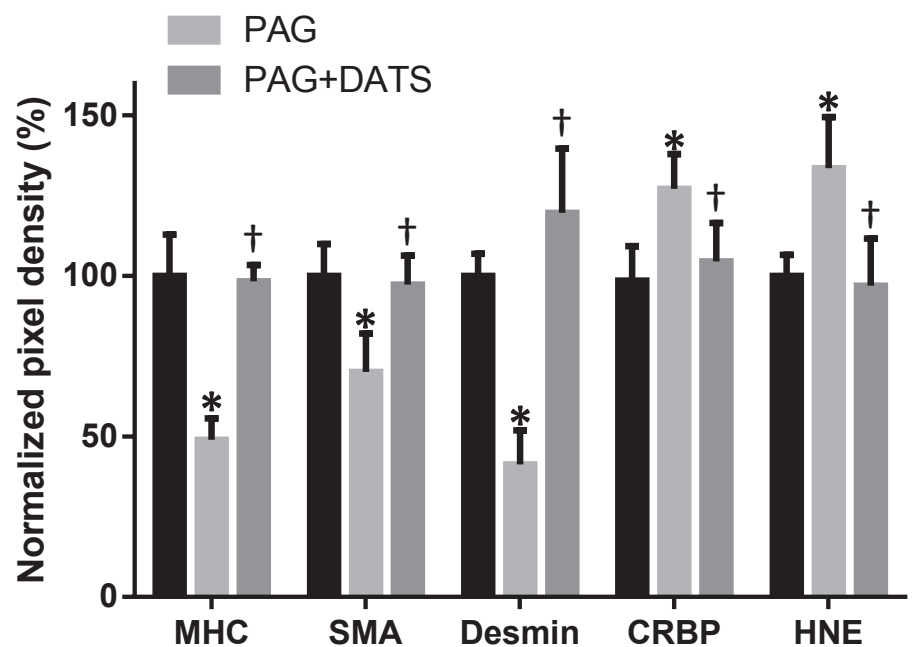

Figure 6 Effect of hydrogen sulfide donor diallyl trisulfide (DATS) on vascular remodeling. Stem villus artery (SVA) explants were challenged with hypoxiareoxygenation (HR; A), or $1 \mathrm{mmol} / \mathrm{L}$ DL-propargylglycine (PAG) under $10 \% \mathrm{O}_{2}$ [normoxia (N); B] in the absence or presence of $500 \mu \mathrm{mol} / \mathrm{L}$ DATS, a long-acting hydrogen sulfide donor derived from garlic, for 3 days. The protein levels of myosin heavy chain (MHC), $\alpha$-smooth muscle actin (SMA), desmin, CRBP, and hydroxynonenal (HNE) in isolated SVAs were quantified by Western blotting. Representative Western blots are shown in the left panels; the average profile in the right panels. $n=5$ (A and $\mathbf{B}) .{ }^{*} P<0.05$ versus normoxic control; ${ }^{\dagger} P<0.05$ versus HR + DATS (A) or PAG + DATS (B) using Tukey's multiple comparison test.

conditions of intraplacental flow predominated in cases of reversed end-diastolic flow. Another morphometric study of placentas from IUGR pregnancies reported a $60 \%$ increase in the thickness of SVA vessel walls, with the wall thickness correlating with the increase in Doppler resistance indexes observed. ${ }^{23}$ However, others reported no significant difference between the diameter distribution of the stem villus arteries between IUGR and age-matched controls. ${ }^{37}$ We found no significant difference in the vessel wall thickness, but report reduced diameter of the lumen and increased wall thickness/lumen ratio. Similarly, increased cell proliferation in the tunica intima and media of the SVAs was demonstrated in preeclamptic pregnancies, which also experience oxidative stress. ${ }^{38}$ Hence, the weight of evidence from this study and from others suggests a correlation between SVA remodeling and placental vascular resistance.

The changes in SVA structure identified by morphometric studies in IUGR closely resemble those described in other forms of vascular injury (eg, atherosclerosis and hypertension). ${ }^{24}$ In these well-studied cases, the thickened tunica media can be accounted for by the dedifferentiation of SMCs, which adopt a synthetic (increased secretion of components of the extracellular matrix) and proliferative (increased SMC number) phenotype. Vascular structural remodeling is considered key to the pathological basis of these diseases. Oxidative stress plays a central role in inducing this phenotype, ${ }^{39,40}$ and is likely to be the underlying pathological process that induces SVA remodeling and consequent increased placental vascular resistance in 
IUGR pregnancies. Doppler changes in the umbilical circulation are invariably seen subsequent to similar changes in the uterine arteries, strongly suggesting that they are a secondary phenomenon to malperfusion of the placenta. We envisage that deficient conversion of the maternal spiral arteries supplying the placenta results in an ischemiareperfusion type injury, and have established an explant model for SVAs to test this assumption. Subjecting SVA explants to HR in vitro for 1 to 3 days resulted in oxidative stress and SMC dedifferentiation, with reduced expression of the contractile proteins and differentiation markers MHC and SMA. Similarly, our data indicate dedifferentiation and vascular remodeling in vivo in the SVAs from IUGR placentas with absent or reduced end-diastolic flow, as shown by reduced expression of MHC, SMA, and desmin, an increase in the dedifferentiation markers CRBP1 and MMP2, and the increased vessel wall/lumen ratio. These data are consistent with our hypothesis that SMC dedifferentiation is the underlying cellular process that drives changes in fetal vascular resistance in pathological pregnancies.

In the same way that oxidative stress may induce SMC dedifferentiation, leading to vascular remodeling, hydrogen sulfide may also modulate SMC differentiation status. Administration of hydrogen sulfide to isolated vascular SMCs leads to an increase in differentiation markers, whereas CSE inhibition results in a decrease in these markers as well as increased proliferation. ${ }^{25}$ Similarly, in an Apoe knockout mouse model of atherosclerosis, aortic hydrogen sulfide production is reduced. Exogenous hydrogen sulfide reduces atherosclerotic plaque size, whereas CSE inhibition causes the reverse, observations that can be explained by hydrogen sulfide promoting a differentiated SMC phenotype. ${ }^{41}$ Consistent with the above findings, we have shown that CSE expression is reduced in vivo in pathological pregnancies, and expression levels are correlated with SMC differentiation status, and ultimately, birth weight. Moreover, in our SVA explant model, we have shown that CSE inhibition under normoxic conditions produces changes in SMC marker expression similar to those induced by HR, and that the dedifferentiated phenotype of HR-treated SVA explants could be prevented by concomitant treatment with the hydrogen sulfide donor DATS. Upstream to CSE, the transcription factor specificity protein-1 is a critical regulator of CSE expression during SMC phenotype change,$^{25}$ and specificity protein- 1 is in turn regulated by miR-21. We have previously demonstrated that miR-21 correlates negatively with CSE expression in placentas with abnormal umbilical artery waveforms, and that exposure of villus explants to HR led to an increase in miR-21 and a significant reduction in CSE protein and mRNA. ${ }^{17}$ Interestingly, CSE inhibition under normoxic conditions increased the level of oxidative stress in SVAs. This may suggest that signaling is two way (ie, oxidative stress reduces hydrogen sulfide signaling, which in turn leads to enhanced oxidative stress). This would imply a positive feedback loop whereby poor placentation, once crossing a certain threshold, would lead to progressive fetoplacental pathology. This could explain, for instance, the increasing umbilical artery pulsatility index seen with advancing gestation in pregnancies complicated by severe IUGR. ${ }^{4}$

Consistent with these roles of hydrogen sulfide, the consumption of garlic is negatively correlated with the progression of cardiovascular disease, and causes a lower incidence of hypertension, enhances antioxidants, and inhibits platelet aggregation. ${ }^{43}$ It has been shown that the biological production of hydrogen sulfide from garlic-derived organic polysulfides mediates the major beneficial effects of garlic-rich diets, specifically on cardiovascular disease and more broadly on overall health. ${ }^{44}$ In animal models of hypoxic pulmonary hypertension, hydrogen sulfide could reverse structural remodeling changes in pulmonary vessels of rats exposed to chronic HR. ${ }^{45}$ Hydrogen sulfide therapy also protected against acute myocardial ischemia/reperfusion, ${ }^{46}$ and attenuated cardiac dysfunction after heart failure. ${ }^{47,48}$ The use of garlic as a preventative therapy for preeclampsia and hypertensive complications of pregnancy has been considered in a Cochrane review in $2006,{ }^{49}$ which concluded there is presently insufficient evidence to recommend increased garlic intake for preventing preeclampsia, and suggested further large randomized clinical trials should be performed. There are several garlic extract preparations (eg, allicin and DATS) available over the counter. These are safe to use during pregnancy, as the Cochrane review concluded, ${ }^{49}$ and thus highly amenable to translational study in a clinical context. DATS was used in our in vitro experiments, where it successfully prevented the smooth muscle dedifferentiation induced by oxidative stress of HR, or by CSE inhibition. We hypothesize that by maintaining differentiated smooth muscle cell status in the fetoplacental circulation, hydrogen sulfide preserves maximal placental blood flow during pregnancy. This, in turn, will promote good fetal growth, and reduce perinatal morbidity and mortality.

In conclusion, we show that the increased placental vascular resistance observed in pregnancies complicated by IUGR is associated with SMC dedifferentiation and SVA remodeling, and with reduced hydrogen sulfide signaling. We further show that oxidative stress, as occurs in poor placentation, is able to induce SVA remodeling, acting principally via reducing hydrogen sulfide signaling. Reduced hydrogen sulfide signaling is necessary and sufficient for SVA remodeling to occur. Given that hydrogen sulfide signaling explains $43 \%$ of the variation in birth weight in this human cohort, this strongly suggests that hydrogen sulfide donors are likely to improve pregnancy outcome and the long-term health of individuals, and this should be studied further in the clinical setting for potential therapeutic use. 


\section{Acknowledgments}

\section{We thank the Cambridge Comprehensive Biomedical Research Centre and the staff of the Rosie Hospital for their help in collecting the normal term placentas.}

\section{References}

1. Barker DJ, Gluckman PD, Godfrey KM, Harding JE, Owens JA, Robinson JS: Fetal nutrition and cardiovascular disease in adult life. Lancet 1993, 341:938-941

2. Ojeda NB, Grigore D, Alexander BT: Intrauterine growth restriction: fetal programming of hypertension and kidney disease. Adv Chronic Kidney Dis 2008, 15:101-106

3. Stocker CJ, Arch JR, Cawthorne MA: Fetal origins of insulin resistance and obesity. Proc Nutr Soc 2005, 64:143-151

4. Meyer K, Lubo Z: Fetal programming of cardiac function and disease. Reprod Sci 2007, 14:209-216

5. Gluckman PD, Hanson MA, Cooper C, Thornburg KL: Effect of in utero and early-life conditions on adult health and disease. N Engl J Med 2008, 359:61-73

6. Arbeille P: Fetal arterial Doppler-IUGR and hypoxia. Eur J Obstet Gynecol Reprod Biol 1997, 75:51-53

7. Soregaroli M, Bonera R, Danti L, Dinolfo D, Taddei F, Valcamonico A, Frusca T: Prognostic role of umbilical artery Doppler velocimetry in growth-restricted fetuses. J Matern Fetal Neonatal Med 2002, 11:199-203

8. Steiner H, Staudach A, Spitzer D, Schaffer KH, Gregg A, Weiner CP: Growth deficient fetuses with absent or reversed umbilical artery enddiastolic flow are metabolically compromised. Early Hum Dev 1995, $41: 1-9$

9. Kingdom J, Huppertz B, Seaward G, Kaufmann P: Development of the placental villous tree and its consequences for fetal growth. Eur J Obstet Gynecol Reprod Biol 2000, 92:35-43

10. Baschat AA: Neurodevelopment following fetal growth restriction and its relationship with antepartum parameters of placental dysfunction. Ultrasound Obstet Gynecol 2011, 37:501-514

11. Redman CW, Sargent IL: Latest advances in understanding preeclampsia. Science 2005, 308:1592-1594

12. Burton GJ, Jauniaux E: Placental oxidative stress: from miscarriage to preeclampsia. J Soc Gynecol Investig 2004, 11:342-352

13. Boura AL, Walters WA: Autacoids and the control of vascular tone in the human umbilical-placental circulation. Placenta 1991, 12:453-477

14. Zhao W, Zhang J, Lu Y, Wang R: The vasorelaxant effect of H(2)S as a novel endogenous gaseous K(ATP) channel opener. EMBO J 2001, 20:6008-6016

15. Coletta C, Papapetropoulos A, Erdelyi K, Olah G, Modis K, Panopoulos P, Asimakopoulou A, Gero D, Sharina I, Martin E, Szabo C: Hydrogen sulfide and nitric oxide are mutually dependent in the regulation of angiogenesis and endothelium-dependent vasorelaxation. Proc Natl Acad Sci U S A 2012, 109:9161-9166

16. Yang G, Wu L, Jiang B, Yang W, Qi J, Cao K, Meng Q, Mustafa AK, Mu W, Zhang S, Snyder SH, Wang R: H2S as a physiologic vasorelaxant: hypertension in mice with deletion of cystathionine gammalyase. Science 2008, 322:587-590

17. Cindrova-Davies T, Herrera EA, Niu Y, Kingdom J, Giussani DA, Burton GJ: Reduced cystathionine gamma-lyase and increased miR-21 expression are associated with increased vascular resistance in growthrestricted pregnancies: hydrogen sulfide as a placental vasodilator. Am J Pathol 2013, 182:1448-1458

18. Wang K, Ahmad S, Cai M, Rennie J, Fujisawa T, Crispi F, Baily J, Miller MR, Cudmore M, Hadoke PW, Wang R, Gratacos E, Buhimschi IA, Buhimschi CS, Ahmed A: Dysregulation of hydrogen sulfide producing enzyme cystathionine gamma-lyase contributes to maternal hypertension and placental abnormalities in preeclampsia. Circulation 2013, 127:2514-2522

19. Hu TX, Wang G, Guo XJ, Sun QQ, He P, Gu H, Huang Y, Gao L, Ni X: MiR 20a,-20b and -200c are involved in hydrogen sulfide stimulation of VEGF production in human placental trophoblasts. Placenta 2016, 39:101-110

20. Holwerda KM, Bos EM, Rajakumar A, Ris-Stalpers C, van Pampus MG, Timmer A, Erwich JJ, Faas MM, van Goor H, Lely AT: Hydrogen sulfide producing enzymes in pregnancy and preeclampsia. Placenta 2012, 33:518-521

21. Fok RY, Pavlova Z, Benirschke K, Paul RH, Platt LD: The correlation of arterial lesions with umbilical artery Doppler velocimetry in the placentas of small-for-dates pregnancies. Obstet Gynecol 1990, 75: $578-583$

22. Salafia CM, Pezzullo JC, Minior VK, Divon MY: Placental pathology of absent and reversed end-diastolic flow in growth-restricted fetuses. Obstet Gynecol 1997, 90:830-836

23. Mitra SC, Seshan SV, Riachi LE: Placental vessel morphometry in growth retardation and increased resistance of the umbilical artery Doppler flow. J Matern Fetal Med 2000, 9:282-286

24. Rzucidlo EM, Martin KA, Powell RJ: Regulation of vascular smooth muscle cell differentiation. J Vasc Surg 2007, 45(Suppl A): A25-A32

25. Yang G, Pei Y, Teng H, Cao Q, Wang R: Specificity protein-1 as a critical regulator of human cystathionine gamma-lyase in smooth muscle cells. J Biol Chem 2011, 286:26450-26460

26. Yang G, Pei Y, Cao Q, Wang R: MicroRNA-21 represses human cystathionine gamma-lyase expression by targeting at specificity protein-1 in smooth muscle cells. J Cell Physiol 2012, 227: $3192-3200$

27. Zhu S, Wu H, Wu F, Nie D, Sheng S, Mo YY: MicroRNA-21 targets tumor suppressor genes in invasion and metastasis. Cell Res 2008, 18: 350-359

28. Yao Q, Xu H, Zhang QQ, Zhou H, Qu LH: MicroRNA-21 promotes cell proliferation and down-regulates the expression of programmed cell death 4 (PDCD4) in HeLa cervical carcinoma cells. Biochem Biophys Res Commun 2009, 388:539-542

29. Cheng Y, Ji R, Yue J, Yang J, Liu X, Chen H, Dean DB, Zhang C: MicroRNAs are aberrantly expressed in hypertrophic heart: do they play a role in cardiac hypertrophy? Am J Pathol 2007, 170: $1831-1840$

30. Naraba H, Iwai N: Assessment of the microRNA system in saltsensitive hypertension. Hypertens Res 2005, 28:819-826

31. Pauly RR, Passaniti A, Bilato C, Monticone R, Cheng L, Papadopoulos N, Gluzband YA, Smith L, Weinstein C, Lakatta EG, Crow MT: Migration of cultured vascular smooth muscle cells through a basement membrane barrier requires type IV collagenase activity and is inhibited by cellular differentiation. Circ Res 1994, 75:41-54

32. Owens GK, Kumar MS, Wamhoff BR: Molecular regulation of vascular smooth muscle cell differentiation in development and disease. Physiol Rev 2004, 84:767-801

33. Hoofnagle MH, Thomas JA, Wamhoff BR, Owens GK: Origin of neointimal smooth muscle: we've come full circle. Arterioscler Thromb Vasc Biol 2006, 26:2579-2581

34. Lausman A, McCarthy FP, Walker M, Kingdom J: Screening, diagnosis, and management of intrauterine growth restriction. J Obstet Gynaecol Can 2012, 34:17-28

35. Cindrova-Davies T, Yung HW, Johns J, Spasic-Boskovic O, Korolchuk S, Jauniaux E, Burton GJ, Charnock-Jones DS: Oxidative stress, gene expression, and protein changes induced in the human placenta during labor. Am J Pathol 2007, 171:1168-1179

36. Jackson MR, Walsh AJ, Morrow RJ, Mullen JB, Lye SJ, Ritchie JW: Reduced placental villous tree elaboration in small-for-gestational-age pregnancies: relationship with umbilical artery Doppler waveforms. Am J Obstet Gynecol 1995, 172:518-525

37. Guiot C, Todros T, Pianta P, Sciarrone A, Kosanke G, Kaufan P: The diameter distribution of the stem villi arteries does not discriminate 
between normal and intra uterine growth restricted placentas. J Theor Med 1999, 1:263-273

38. Las Heras J, Haust MD, Harding PG: Morphology of fetal placental stem arteries in hypertensive disorders ("toxemia") of pregnancy. Appl Pathol 1983, 1:301-309

39. Irani K: Oxidant signaling in vascular cell growth, death, and survival: a review of the roles of reactive oxygen species in smooth muscle and endothelial cell mitogenic and apoptotic signaling. Circ Res 2000, 87: 179-183

40. Sung HJ, Eskin SG, Sakurai Y, Yee A, Kataoka N, McIntire LV: Oxidative stress produced with cell migration increases synthetic phenotype of vascular smooth muscle cells. Ann Biomed Eng 2005, 33:1546-1554

41. Mani S, Li H, Untereiner A, Wu L, Yang G, Austin RC, Dickhout JG, Lhotak S, Meng QH, Wang R: Decreased endogenous production of hydrogen sulfide accelerates atherosclerosis. Circulation 2013, 127: 2523-2534

42. Baschat AA, Gembruch U, Harman CR: The sequence of changes in Doppler and biophysical parameters as severe fetal growth restriction worsens. Ultrasound Obstet Gynecol 2001, 18:571-577

43. Banerjee SK, Maulik SK: Effect of garlic on cardiovascular disorders: a review. Nutr J 2002, 1:4
44. Benavides GA, Squadrito GL, Mills RW, Patel HD, Isbell TS, Patel RP, Darley-Usmar VM, Doeller JE, Kraus DW: Hydrogen sulfide mediates the vasoactivity of garlic. Proc Natl Acad Sci U S A 2007, 104:17977-17982

45. Hongfang J, Cong B, Zhao B, Zhang C, Liu X, Zhou W, Shi Y, Tang C, Junbao D: Effects of hydrogen sulfide on hypoxic pulmonary vascular structural remodeling. Life Sci 2006, 78:1299-1309

46. Calvert JW, Jha S, Gundewar S, Elrod JW, Ramachandran A, Pattillo CB, Kevil CG, Lefer DJ: Hydrogen sulfide mediates cardioprotection through Nrf2 signaling. Circ Res 2009, 105:365-374

47. Polhemus D, Kondo K, Bhushan S, Bir SC, Kevil CG, Murohara T, Lefer DJ, Calvert JW: Hydrogen sulfide attenuates cardiac dysfunction following heart failure via induction of angiogenesis. Circ Heart Fail 2013, 6:1077-1086

48. Kondo K, Bhushan S, King AL, Prabhu SD, Hamid T, Koenig S, Murohara T, Predmore BL, Gojon G Sr, Gojon G Jr, Wang R, Karusula N, Nicholson CK, Calvert JW, Lefer DJ: H(2)S protects against pressure overload-induced heart failure via upregulation of endothelial nitric oxide synthase. Circulation 2013, 127:1116-1127

49. Meher S, Duley L: Garlic for preventing pre-eclampsia and its complications. Cochrane Database Syst Rev 2006:CD006065 